\title{
Detection of crystalline silicates around the T Tauri star Hen $3-600 \mathrm{~A}^{1}$
}

\author{
Mitsuhiko Honda ${ }^{2,3}$, Hirokazu Kataza ${ }^{4}$, Yoshiko K. Okamoto ${ }^{5}$, Takashi Miyata ${ }^{6}$, Takuya \\ Yamashita $^{3,2}$, Shigeyuki Sako ${ }^{2,3}$, Shinya Takubo ${ }^{2,3}$ and Takashi Onaka ${ }^{2}$
}

\begin{abstract}
We have carried out mid-infrared N-band spectroscopic observations of the T Tauri star Hen 3-600A in the TW Hydra association with the COMICS on the $8.2 \mathrm{~m}$ Subaru Telescope and found structured features in its spectrum. These structured features are well explained by a combination of crystalline forsterite, crystalline enstatite, silica and glassy olivine grains. Among intermediate-mass young stellar objects (YSOs), crystalline silicates have already been detected, but no firm detection has been reported so far for low-mass YSOs such as T Tauri stars. This is the first clear detection of crystalline silicates in low-mass YSOs and shows that the crystallization event occurs even in the protoplanetary disk of low-mass YSOs in the T Tauri phase. The physical processes leading to the inferred dust composition in the Hen3-600A system may be analogous to those occured in the early epoch of the Solar system.
\end{abstract}

Subject headings: circumstellar matter — stars: pre-main sequence

\footnotetext{
${ }^{1}$ Based on data collected at Subaru Telescope, which is operated by the National Astronomical Observatory of Japan.

${ }^{2}$ Department of Astronomy, School of Science, University of Tokyo, Bunkyo-ku, Tokyo 113-0033, Japan, onaka@astron.s.u-tokyo.ac.jp

${ }^{3}$ Subaru Telescope, National Astronomical Observatory of Japan, 650 North A'ohoku Place, Hilo, Hawaii 96720, U.S.A., hondamt@subaru.naoj.org, takuya@subaru.naoj.org,sako@subaru.naoj.org

${ }^{4}$ Center for Advanced Spacecraft Technology, Institute of Space and Astronautical Science, 3-1-1 Yoshinodai, Sagamihara, Kanagawa 229-8510, Japan, kataza@ir.isas.ac.jp

${ }^{5}$ Institute of Physics, Center for Natural Science, Kitasato University 1-15-1 Kitasato, Sagamihara, Kanagawa 229-8555, Japan, okamtoys@cc.nao.ac.jp

${ }^{6}$ Kiso Observatory, Institute of Astronomy, School of Science, University of Tokyo, Mitake, Nagano $397-$ 0101, Japan, miyata@kiso.ioa.s.u-tokyo.ac.jp
} 


\section{Introduction}

Silicate is one of the main components of dust in the Universe. In the interstellar medium (ISM), it is believed to be completely amorphous as implicated by the spectra with broad silicate absorption features and no evidence for crystalline silicate has been indicated in the ISM. Since the ISM material is the starting material for the star formation, the presence of amorphous silicate in most YSOs is a natural consequence. However, some Herbig Ae/Be stars (Hanner et al. 1995; Malfait et al. 1998; Sitko et al. 1999; Bouwman et al. 2001) and the Vega-like star $\beta$ Pic (Knacke et al. 1993) exhibit the spectra which clearly deviates from the amorphous silicate features. Their spectra show crystalline silicate features and indicate the presence of large grains, which demonstrates dust processing in the protoplanetary disks. By studying the dust composition of these YSOs, one can derive the physical processes occurring in, and the evolution of the circumstellar disks. In addition, these YSO spectra resemble cometary spectra. Comets often shows crystalline silicates (Hanner et al. 1994; Crovisier et al. 1997), preserving the record of the dust processing in the early phase of the proto solar nebula, suggesting that similar processes occurred in the early history of the solar system as are now observed in the YSOs.

However, most studies of dust processing so far made for YSOs are limited to Herbig Ae/Be stars, which might be similar to, but are obviously more massive than the solar-type stars. It is thus important to investigate the dust processing in T Tauri stars, which are supposed to be a good example of the early stage of the solar-type stars, to understand the history of the solar system. To date, among low-mass YSOs, there is no firm detection of crystalline silicates, which is a typical indicater of dust processing. Most mid-infrared spectroscopic studies of dust in low-mass YSOs showed the amorphous silicate emission or absorption features (e.g. Natta et al. 2000). Hanner et al. (1995, 1998) also concluded that the amorphous silicate emission accounts for their observed emission features of $\mathrm{T}$ Tauri stars and FU Ori stars and that the $11.2 \mu \mathrm{m}$ feature seen in some objects can be attributed to polycyclic aromatic hydrocarbon (PAH) emission. On the other hand, an unusually broad silicate emission feature was reported for HD98800 (K5V; Skinner et al. 1992; Sylvester et al. 1996), a 10 Myr old pre-main sequence star (PMS) in the TW Hya Association (TWA). Such a broad silicate emission feature was thought to be the evidence for dust growth (Skinner et al. 1992; Sylvester et al. 1996), while Koener et al. (2000) claimed that crystalline silicates may account for it. TW Hya (K7Ve) is also a member of TWA, and is assumed to be an old classical T Tauri star (CTTS). Its mid-infrared spectrum was first presented by Sitko et al. (2000). They could not detect the crystalline olivine feature at $11.2 \mu \mathrm{m}$, and concluded that its silicate emission feature is similar to many other young pre-main sequence stars. However, Weinberger et al. (2002) claimed hints for a crystalline olivine feature at $11.2 \mu \mathrm{m}$ in their N-band spectrum of TW Hya with a high S/N ratio and spectral resolution $(\mathrm{R} \sim 120)$. 
Since unusual silicate emission features were reported for old low-mass YSOs, such as TW Hya and HD98800, it would be a good strategy to investigate old low-mass YSOs from the view point of silicate dust evolution. However, most old low-mass YSOs tend to be faint in the mid-infrared. Efforts to search for the evidence for crystalline silicates in low-mass YSOs have so far been hampered by the faintness of the objects. The COoled Mid-Infrared Camera and Spectrometer (COMICS) on the $8.2 \mathrm{~m}$ Subaru Telescope provides the higher sensitivity and enough spectral resolution $(\mathrm{R} \sim 250)$ to solve this issue. Therefore, we carried out the mid-infrared spectroscopic survey of low-mass YSOs, focusing on relatively old (5 10 Myr) objects.

In this Letter, we present the results of mid-infrared N-band spectroscopic observations of Hen3-600 from our mid-infrared spectroscopic survey with COMICS. It is a multiple system consisting of the spectroscopic binary primary star A (M3) and the secondary star B (M3.5), with a separation of $1.4^{\prime \prime}$. It is a member of the TWA, whose age is estimated to be 1 10Myr old (see discussions of Jayawardhana et al. 1999). Muzerolle et al. (2000) derived the mass accretion rate of Hen3-600A to be $5 \times 10^{-11} \mathrm{M}_{\odot} \mathrm{yr}^{-1}$ from optical spectroscopy, which is by 1-2 orders of magnitude lower than the average rate in one-Myr old objects, suggesting the significant disk evolution and aging of the system. Mid-infrared imaging observations by Jayawardhana et al. (1999) showed that the the primary A has most of the circumstellar dust emission in this system. They also pointed out a possibility of the inner hole in the circumstellar disk from the SED of Hen3-600A. The present letter reports the clear detection of crystalline silicate features in old low-mass YSOs for the first time.

\section{Observations and Data Analysis}

We observed Hen 3-600 with the COoled Mid-Infrared Camera and Spectrometer (COMICS; Kataza et al. 2000; Okamoto et al. 2002) mounted on the 8.2m SUBARU Telescope on Dec 27 2001. COMICS is an instrument for imaging and long-slit spectroscopy in the 10 and $20 \mu \mathrm{m}$ atmospheric windows. N-band low-resolution ( $\mathrm{R} \sim 250)$ spectroscopic observations were performed with a $0.33^{\prime \prime}$ wide slit that was set to run through Hen3-600A and B (The position angle was $\left.215^{\circ}\right)$. Imaging observations in the $8.8 \mu \mathrm{m}(\Delta \lambda=0.8 \mu \mathrm{m})$ and $12.4 \mu \mathrm{m}(\Delta \lambda=1.2 \mu \mathrm{m})$ bands were also made for the calibration of the absolute flux of the spectra. To cancel the high background radiation, the secondary mirror chopping was used at a frequency of $0.50 \mathrm{~Hz}$ with a 10 " throw and a direction to the position angle of $215^{\circ}$. The total on-source integration time was 598sec. We selected HD123123 (Cohen et al. 1999) as a rationing star for the correction of the atmospheric absorption and a flux standard star for aperture photometry. The observation parameters are summarized in Table 1. 
For the data reduction, we used our own reduction tools and IRAF. For the imaging data, the standard reduction procedure and aperture photometry were applied. For the spectroscopic data, the standard chopping subtraction and flat-fielding by thermal spectra of the telescope cell-cover were made and the distortion of the spectral image on the detector was corrected. Then the spectra of Hen3-600A and of HD123123 were extracted. Due to the low chopping frequency, sky emission was not completely canceled out. Therefore we derived the blank sky average level from the same object frame, and subtracted it as an offset from each extracted spectrum. The wavelength was calibrated by atmospheric emission lines. After we divided the Hen3-600A spectrum by the HD123123 spectrum for the correction of atmospheric absorption, we multiplied the resultant spectrum by the template of HD123123 (Cohen et al. 1999). Finally we calibrated the absolute flux of the spectrum of Hen3-600A by the photometric data in the $8.8 \mu \mathrm{m}$ and $12.4 \mu \mathrm{m}$ bands. The statistical uncertainty in the flux at each wavelength was estimated from the noise in the blank sky and shown in Figure 1. The ozone absorption has increased the uncertainty in $9.3-10.0 \mu \mathrm{m}$ due to the airmass mismatch between the objects and the standards. We estimate the uncertainty at the wavelength of strongest ozone absorption $(9.6 \mu \mathrm{m})$ to be at most $7 \%$.

\section{Results}

Figure 1 shows the observed 8-13 $\mu$ m spectrum of Hen 3-600A. A broad silicate emission feature is clearly seen, whose profile is much broader than that of amorphous silicate frequently seen for a variety of astronomical objects. In addition, it shows multiple local peaks located at $9.2 \mu \mathrm{m}, 10.1 \mu \mathrm{m}, 10.5 \mu \mathrm{m}, 10.9 \mu \mathrm{m}, 11.2 \mu \mathrm{m}$ and $12.5 \mu \mathrm{m}$.

To analyze it further, we made the following spectral model fitting. We considered 4 dust species in our spectral model as described below. The spectral profiles of these dust species are presented in Figure 2.

1. Glassy olivine: For a representative of amorphous silicate, which is often observed in the spectra of many YSOs, we used the optical constants of glassy olivine by Dorschner et al. (1995). Amorphous olivine grains show little dependence on the grain shape, however, it depends on grain size (Bouwman et al. 2001). To take grain size effect into account, we calculated the mass-absorption coefficient using the Mie theory for $0.1 \mu \mathrm{m}$ and $2.0 \mu \mathrm{m}$ spherical particles (van de Hulst 1957; Matsumura \& Seki 1986). The $0.1 \mu \mathrm{m}$ glassy olivine causes a narrow structure-less feature peaking at $9.8 \mu \mathrm{m}$ in the $10 \mu \mathrm{m}$ region, while that of the $2.0 \mu \mathrm{m}$ has a broad trapezoidal structure-less feature (see Figure 2). 
2. Crystalline forsterite: For the candidate for the observed structured features, we used the mass-absorption coefficient of crystalline forsterite (Koike et al. 2000a). In the 8$13 \mu \mathrm{m}$ region, forsterite has emissivity peaks at $10.06 \mu \mathrm{m}, 10.42 \mu \mathrm{m}, 11.24 \mu \mathrm{m}$ (strong), and $11.89 \mu \mathrm{m}$.

3. Crystalline orthoenstatite: Pyroxene is one of the typical silicates as well as olivine. We used the mass-absorption coefficient of crystalline orthoenstatite (Koike et al. 2000b). Crystalline orthoenstatite of synthetic pure material has emissivity peaks at $9.34 \mu \mathrm{m}$, $9.87 \mu \mathrm{m}, 10.70 \mu \mathrm{m}$, and $11.68 \mu \mathrm{m}$ in the $10 \mu \mathrm{m}$ region. Both the optical data of forsterite and orthoenstatite were obtained by the absorption measurements of small particles. Their shapes are rather random and may be represented by the continuous distribution of ellipsoids (Koike et al., private communication).

4. Silica: Laboratory studies show that thermal annealing transforms the amorphous $\mathrm{Mg}$ silicate smokes into crystalline forsterite and crystalline/amorphous $\mathrm{SiO}_{2}$ (Fabian et al. 2000). We take into account silica and use the parameters of Spitzer \& Kleinman (1961). The optical profile of silica is very sensitive to the grain shape. Small spherical particles can not account for the observed spectrum. We have therefore calculated the mass-absorption coefficient by assuming a continuous distribution of ellipsoids (CDE; e.g. Bohren \& Huffman 1983) in the Rayleigh limit. The calculated mass-absorption coefficient has peaks at $\sim 9.1 \mu \mathrm{m}$ and $\sim 12.5 \mu \mathrm{m}$.

We did not consider PAH emission because PAH emission should be associated with the features at $7.7 \mu \mathrm{m}, 8.6 \mu \mathrm{m}, 11.3 \mu \mathrm{m}$, and $12.7 \mu \mathrm{m}$, which are not detected for Hen3-600A. The late spectral type (M3) of Hen3-600A is also consistent with the absence of PAH emission because it cannot supply enough UV photons to enhance PAH emission. Therefore, we consider only above 4 dust species in the following analysis.

In the model fit, we modified a case 2 model of Hanner et al. (1995, 1998). The model flux $F_{\lambda}$ consists of two components, featureless power-law continuum emission and optically thin emission of the dust features. A power-law source function is assumed. The model spectrum can be written by

$$
\lambda F_{\lambda}=a_{0}\left(\frac{\lambda}{9.8 \mu m}\right)^{n}+\left(a_{1} Q_{a 0.1}+a_{2} Q_{a 2.0}+a_{3} Q_{\text {forst }}+a_{4} Q_{\text {ensta }}+a_{5} Q_{\text {silica }}\right)\left(\frac{\lambda}{9.8 \mu m}\right)^{m},
$$

where $Q_{a 0.1}, Q_{a 2.0}, Q_{\text {forst }}, Q_{\text {ensta }}$, and $Q_{\text {silica }}$ are the absorption efficiencies of $0.1 \mu \mathrm{m}$ and $2.0 \mu \mathrm{m}$ glassy olivine, crystalline forsterite, crystalline enstatite, and silica, respectively. They are shown in Figure 2. There are 8 free parameters $\left(6\right.$ multiplication factors $a_{0}, a_{1}, a_{2}, a_{3}, a_{4}$ in $10^{-14} \mathrm{Wm}^{-2}$ and 2 spectral indices of source function, $n$ and $m$ ). We employed a leastsquares minimization method to determine the most likely parameters. Resulting best-fit 
parameters are $a_{0}=1.8, a_{1}=0.4, a_{2}=0.0, a_{3}=0.3, a_{4}=0.2, a_{5}=0.4, n=0.2$, and $m=0.5$. The best-fit model spectrum using the derived parameters are shown in Figure 3.

Figure 3 indicates that the $10 \mu \mathrm{m}$ spectrum of Hen 3-600A is well reproduced by the model spectrum. The peaks at $10.1 \mu \mathrm{m}, 10.5 \mu \mathrm{m}$, and $11.2 \mu \mathrm{m}$ are attributed to forsterite. The $12.5 \mu \mathrm{m}$ and $9.2 \mu \mathrm{m}$ peaks can be owing to silica. The $10.9 \mu \mathrm{m}$ peak is likely to come from orthoenstatite, though the observed peak is slightly different from the orthoenstatite peak at $10.7 \mu \mathrm{m}$. Other orthoenstatite features are not seen in the spectrum or located at the wavelengths that are disturbed by atmospheric ozone. Fe bearing pyroxene species can not account for the peak shift (Chihara et al. 2002). However, orthoenstatite is needed to fit the overall shape of model spectrum to the observed spectrum and as a candidate for the observed $10.9 \mu \mathrm{m}$ feature. The best-fit result indicates that no $2.0 \mu \mathrm{m}$ glassy olivine is required in the fitting and thus that the particle size effects do not account for the observed band shape. Based on these results, we conclude that a combination of glassy olivine, crystalline forsterite, silica and possibly crystalline orthoenstatite accounts for the observed spectrum.

\section{Discussion}

At present no evidence for crystalline silicates in the ISM has been found, and also the dust around many YSOs appears to be amorphous. Crystalline silicate features have so far been detected only in the intermediate mass YSOs, Herbig Ae/Be stars (Hanner et al. 1995; Malfait et al. 1998; Sitko et al. 1999; Bouwman et al. 2001), while no firm detection of crystalline silicates in $\mathrm{T}$ Tauri stars has been made to date. Our detection of crystalline silicate features in the spectrum of the $\mathrm{T}$ Tauri star Hen 3-600A indicates that the crystallization process does occur even in the circumstellar disk of a low-mass YSO in the T Tauri phase. Hen 3-600A is a TW Hya association member, and probably an old CTTS evolving into a WTTS. Other TWA members, such as TW Hya and HD98800, are also reported to show unusually broad silicate features, which indicate silicate dust evolution (Skinner et al. 1992; Weinberger et al. 2002). Among low-mass YSOs, silicate dust evolution is implicated only for objects in TWA. These results may be attributed to the old age of TWA members, or they may also be the characteristics specific to TWA. It is reported that crystalline silicate grains tend to be present in old YSOs (old Herbig Ae/Be stars)

for intermediate-mass YSOs (Sitko et al. 1999). The present observations suggest that this trend may also hold for low-mass YSOs. A possible simple explanation for this tendency is that it takes a long time to produce an enough amount of crystalline silicate grains to be observable. Alternatively, the crystallization process starts when a YSO gets old enough. 
Further observational studies of a large sample are needed to investigate this tendency.

From our fitting results of the observed features of Hen 3-600A, we conclude that there are at least 4 dust species of glassy olivine, crystalline forsterite, silica, and possibly crystalline enstatite. Crystalline forsterite is a major component in the crystalline silicate observed to date. The presence of $\mathrm{SiO}_{2}$ is expected based on the laboratory thermal annealing experiment (Thompson et al. 2001, 2002; Fabian et al. 2000; Hallenbeck et al. 2000; Rietmeijer et al. 2002) and recent studies of Herbig Ae/Be by Bouwman et al. (2001). The presence of enstatite in the YSO is a matter of debate. Its presence is rare in the YSO (Bouwman et al. 2001), though it is more commonly found around the evolved stars. In this paper, we attribute the $10.9 \mu \mathrm{m}$ feature of the Hen 3-600A spectrum to enstatite and it provides acceptable fitting results. However, Thompson et al. (2002) claimed that the $10 \mu \mathrm{m}$ "enstatite" features are not sufficient evidence to confirm the presence of crystalline enstatite grains. While enstatite is so far the most favorite candidate to account for the observed $10 \mu \mathrm{m}$ features, further investigations are clearly needed to confirm the presence of enstatite in Hen 3-600A. The derived mass ratio of these dust species (glassy olivine, forsterite, enstatite and silica) is estimated to be 14:10:7:2, assuming the same source function and mass distribution. Therefore crystalline silicate components consist of about $50 \%$ (forsterite $30 \%$, enstatite $20 \%$ ) of the total small silicate grains in the optically thin emitting region. The derived mass ratio of forsterite and silica is consistent with the studies of Herbig Ae/Be by Bouwman et al. (2001), which provided the constraint on the composition and structure of the amorphous material.

Low-mass YSOs are progenitors of our Solar system and crystalline silicate grains are probably produced by thermal annealing processes in the circumstellar disks. In the early Solar system, there is evidence for grain heating, such as the formation of chondrules found in primordial meteorites (Jones et al. 2000). Chondrules are thought to be made by flush heating in the solar nebula. There are several models for the formation of chondrules, such as nebular lightening, shock heating, and X-wind models (Jones et al. 2000). Such processes may also lead to annealing of amorphous silicate and prompt silicate dust crystallization. The present detection of crystalline silicates in the T Tauri star Hen3-600A suggests such heating process in the early Solar system may be common among low-mass YSOs.

We are grateful to all of the staff members of SUBARU Telescope for their support. We also thank Drs. Chiyoe Koike, Hiroki Chihara, Hiroshi Suto, and Koji Kawabata for providing us crystalline silicates spectra, calculation codes, and useful comments. Finally, we would like to express thanks to the referee Dr. J. Bouwman for helpful comments. 


\section{REFERENCES}

Bohren, C. F., \& Huffman, D. R. 1983, Absorption and Scattering of Light by Small Particles (John Wily \& Sons Inc.)

Bouwman, J., Meeus, G., de Koter, A., Hony, S., Dominik, C., \& Waters L. B. F. M. 2001, A\&A, 375, 950

Chihara, H., Koike, C., Tsuchiyama, A., \& Tachibana, S. 2002, A\&A, 391, 267

Cohen, M., Walker, R. G., Carter, B., Hammersley, P., Kidger, M., \& Noguchi, K. 1999, ApJ, 117, 1864

Crovisier, J., Leech, K., Bockelee-Morvan, D., Brooke, T. Y., Hanner, M. S., Altieri, B., Keller, H. U., \& Lellouch, E. 1997, Science, 275, 1904

Dorschner, J., Begemann, B., Henning, Th., Jäger, C., \& Mutschke, H. 1995, A\&A, 300, 503

Fabian, D., Jäger, C., Henning, Th., Dorschner, J., \& Mutschke, H. 2000, A\&A, 364, 282

Hallenbeck, S. L., Nuth III, J. A., \& Nelson, R. N. 2000, ApJ, 535, 247

Hanner, M. S., Lynch, D. K., \& Russell, R. W. 1994, ApJ, 425, 274

Hanner, M. S., Brooke, T. Y., \& Tokunaga, A. T. 1995, ApJ, 438, 250

Hanner, M. S., Brooke, T. Y., \& Tokunaga, A. T. 1998, ApJ, 502, 871

Jayawardhana, R., Hartmann, L., Fazio, G., Fisher, R. S., Telesco, C. M., \& Piña, R. K. 1999, ApJ, 520, L41

Jones, R. H., Lee, T., Connolly, H. C., JR., Love, S. G., \& Shang, H. 2000, in Protostars \& Planets IV, ed. Mannings, V., Boss, A. P., \& Russell, S. S. (Tuscon: Univ. Arizona Press)

Kataza, H., Okamoto, Y., Takubo, S., Onaka, T., Sako, S., Nakamura, K., Miyata, T., \& Yamashita, T. 2000, Proc. SPIE, 4008, 1144

Knacke, R. F., Fajardo-Acosta, S. B., Telesco, C. M., Hackwell, J. A., Lynch, D. K., \& Russell, R. W. 1993, ApJ, 418, 440

Koener, D. W., Jensen, E. L. N., Cruz, K. L., Guild, T. B., \& Gultekin, K. 2000, ApJ, 533, L37 
Koike, C., Chihara, H., Tsuchiyama, A., Sogawa, H., \& Suto, H. 2000a, in Proceedings of the 33rd Lunar \& Planetary Symposium,95

Koike, C., Tsuchiyama, A., Shibai, H., Suto, H., Tanabe, T., Chihara, H., Sogawa, H., Mouri, H., \& Okada, K. 2000b, A\&A, 363, 1115

Malfait, K., Waelkens, C., Waters, L. B. F. M., Vandenbussche, B., Huygen, E., \& de Graauw, M. S. 1998, A\&A, 332, L25

Matsumura, M., \& Seki, M. 1986, Ap\&SS, 126, 155

Muzerolle, J., Calvet, N., Briceno, C., Hartmann, L., \& Hillenbrand, L. 2000, ApJ, 535, L47

Natta, A., Meyer, M. R., \& Beckwith, S. V. W. 2000, ApJ, 534, 838

Okamoto, Y. K., Kataza, H., Yamashita, T., Miyata, T., Sako, S., Takubo, S., Honda, M., \& Onaka, T. 2002, Proc. of SPIE, 4841 in press

Rietmeijer, F. J. M., Hallenbeck, S. L., Nuth III, J. A., \& Nelson, R. N. 2002, Icarus, 156, 269

Sitko, M. L., Grady, C. A., Lynch, D. K., Russel, R. W., \& Hanner, M. S., 1999, ApJ, 510, 408

Sitko, M. L., Lynch, D. K.,\& Russel, R. W. 2000, ApJ, 120, 2609

Skinner, C. J., Barlow, M. J., \& Justtanont, K. 1992, MNRAS, 255, 31

Spitzer, W. G. \& Kleinman, D. A. 1961, Phys. Rev. , 121, 1324

Sylvester, R. J., \& Skinner, C. J. 1996, MNRAS, 283, 457

Thompson, S. P., \& Tang, C. C. 2001, A\&A, 368, 721

Thompson, S. P., Fonti, S., Verrienti, C., Blanco, A., Orofino, V., \& Tang, C. C. 2002, A\&A, 395,705

van de Hulst, H.C. 1957, Light Scattering by Small Particles (New York: Wiley)

Weinberger, A. J., Becklin, E. E., Schneider, G., Chiang, E. I., Lowrance, P. J., Silverstone, M., Zuckerman, B., Hines, D. C., \& Smith, B. A. 2002, AJ, 71, 276 


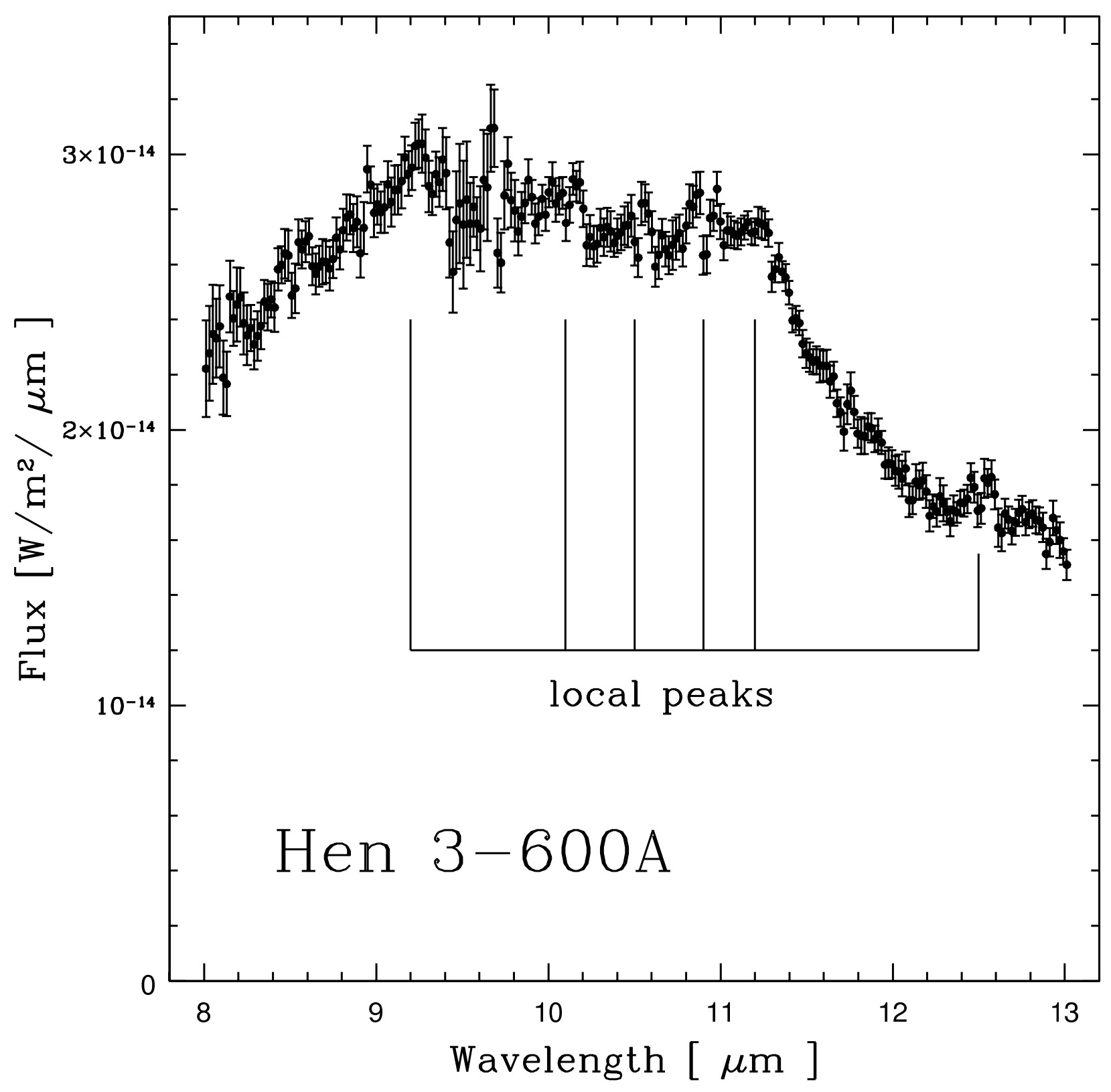

Fig. 1.- Observed N-band spectrum of Hen3-600A. Local peaks at $9.2 \mu \mathrm{m}, 10.1 \mu \mathrm{m}, 10.5 \mu \mathrm{m}$, $10.9 \mu \mathrm{m}, 11.2 \mu \mathrm{m}$, and $12.5 \mu \mathrm{m}$ are indicated by straight lines. 


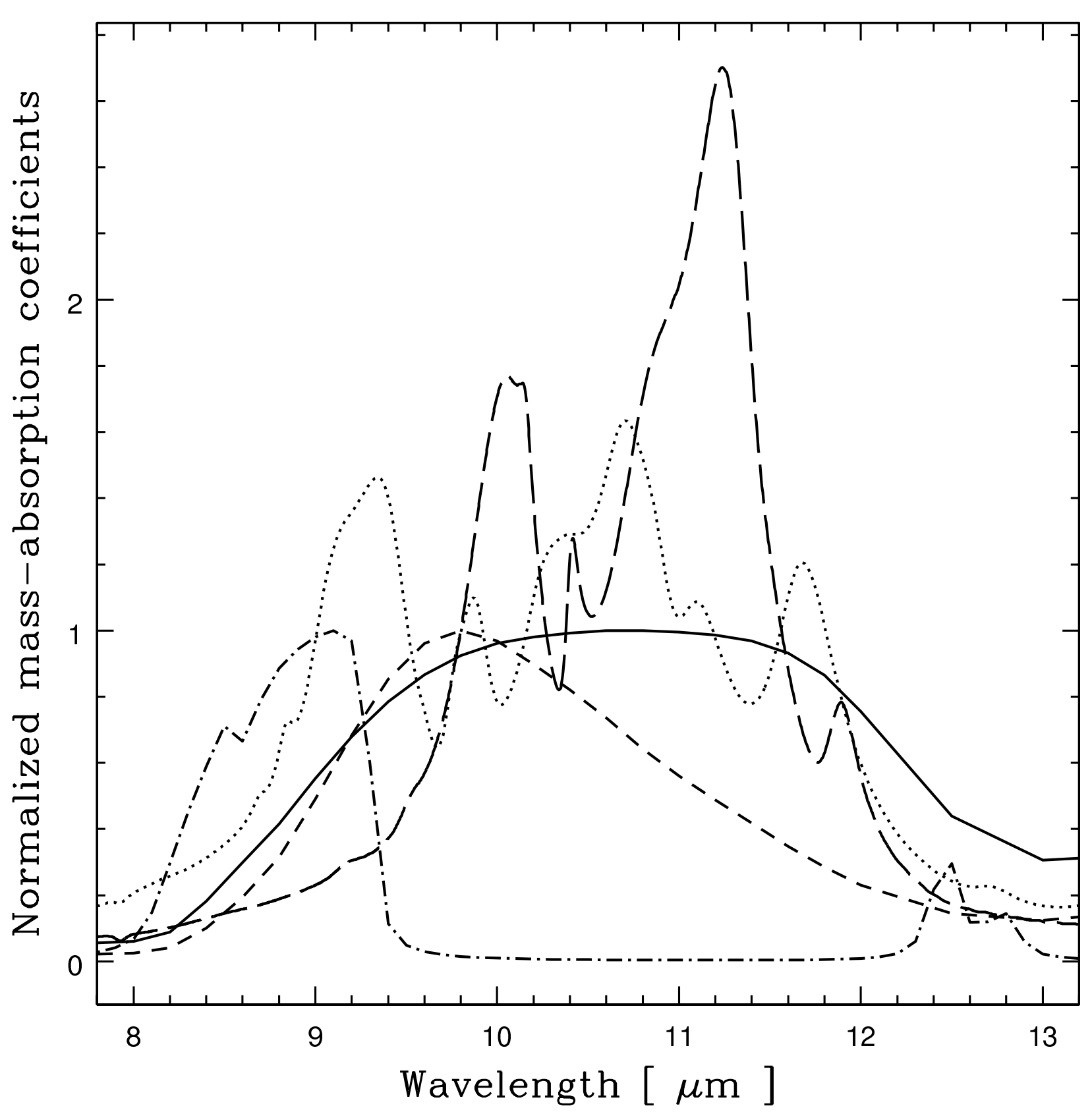

Fig. 2.- Silicate dust spectral profiles of $0.1 \mu \mathrm{m}$ and $2.0 \mu \mathrm{m}$ glassy olivine (dashed line, solid line; Dorschner et al. 1995), crystalline orthoenstatite (dotted line; Koike et al. 2000b), crystalline forsterite (long-dashed line; Koike et al. 2000a), and silica (dot-dashed line; Spitzer \& Kleinman 1961). The normalizing constants in $\mathrm{cm}^{2} \mathrm{~g}^{-1}$ for the $0.1 \mu \mathrm{m}$ and $2.0 \mu \mathrm{m}$ glassy olivine, orthoenstatite, forsterite, and silica are 2372.3, 1664.6, 2451.9, 2476.6, and 16887.2, respectively. 


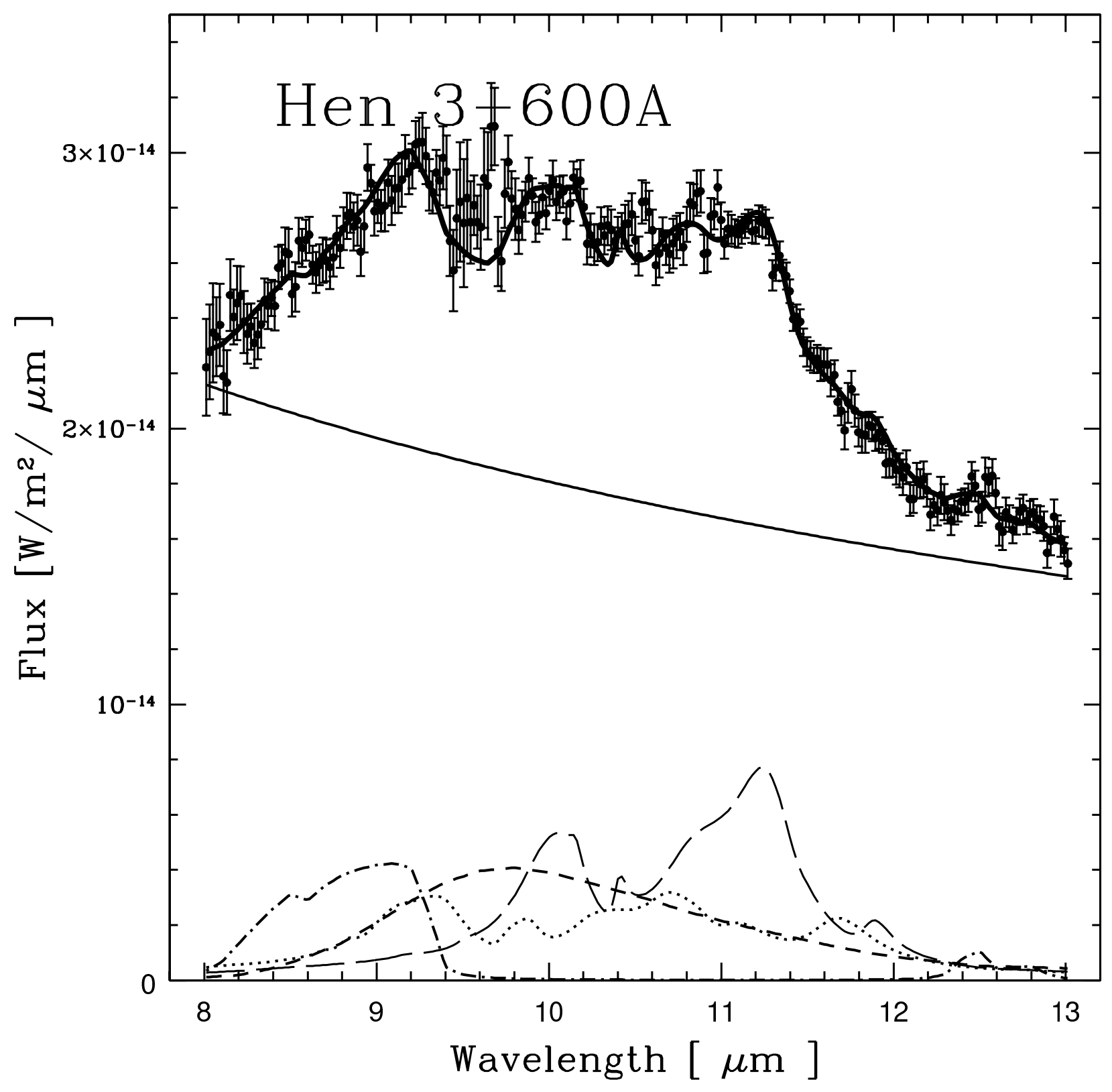

Fig. 3.- Fit result of Hen3-600A. The thick solid line is the best-fit model spectrum, which is the sum of power-law continuum (thin solid), glassy olivine (dashed), orthoenstatite (dotted), forsterite (long-dashed), and silica (dot-dashed). 
Table 1. COMICS Observations of Hen3-600

\begin{tabular}{ccccc}
\hline \hline Mode & Object & Filter $[\mu \mathrm{m}]$ & Integ. Time[sec] & Air Mass \\
\hline \multirow{2}{*}{ Imaging } & Hen 3-600 & $8.8(\Delta \lambda=0.8)$ & 25.9 & 1.879 \\
& Hen 3-600 & $12.4(\Delta \lambda=1.2)$ & 29.6 & 1.855 \\
& HD123123 & $8.8(\Delta \lambda=0.8)$ & 5.1 & 1.975 \\
& HD123123 & $12.4(\Delta \lambda=1.2)$ & 3.2 & 1.754 \\
Spectroscopy & Hen 3-600 & - & 598 & $1.866-1.850$ \\
& HD123123 & - & 40.2 & 1.769 \\
\hline
\end{tabular}

